\title{
DATS - Data Containers for Web Applications
}

\author{
Casen Hunger ${ }^{*}$ \\ University of Texas at Austin \\ casen.h@utexas.edu
}

\author{
Lluís Vilanova \\ Technion - Israel Institute of \\ Technology \\ vilanova@technion.ac.il
}

\author{
Charalampos Papamanthou \\ University of Maryland (UMD) \\ cpap@umd.edu
}

\author{
Yoav Etsion \\ Technion - Israel Institute of \\ Technology \\ yetsion@tce.technion.ac.il
}

\author{
Mohit Tiwari \\ University of Texas at Austin \\ tiwari@austin.utexas.edu
}

\begin{abstract}
Data containers enable users to control access to their data while untrusted applications compute on it. However, they require replicating an application inside each container compromising functionality, programmability, and performance.

We propose DATS - a system to run web applications that retains application usability and efficiency through a mix of hardware capability enhanced containers and the introduction of two new primitives modeled after the popular model-view-controller (MVC) pattern. (1) DATS introduces a templating language to create views that compose data across data containers. (2) DATS uses authenticated storage and confinement to enable an untrusted storage service, such as memcached and deduplication, to operate on plain-text data across containers. These two primitives act as robust declassifiers that allow DATS to enforce non-interference across containers, taking large applications out of the trusted computing base (TCB).

We showcase eight different web applications including Gitlab and a Slack-like chat, significantly improve the worstcase overheads due to application replication, and demonstrate usable performance for common-case usage.
\end{abstract}

CCS Concepts - Security and privacy $\rightarrow$ Authentication; Access control; Authorization; Web application security; Operating systems security; Information flow control;

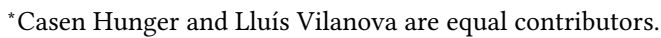

Permission to make digital or hard copies of all or part of this work for personal or classroom use is granted without fee provided that copies are not made or distributed for profit or commercial advantage and that copies bear this notice and the full citation on the first page. Copyrights for components of this work owned by others than the author(s) must be honored. Abstracting with credit is permitted. To copy otherwise, or republish, to post on servers or to redistribute to lists, requires prior specific permission and/or a fee. Request permissions from permissions@acm.org. ASPLOS'18, March 24-28, 2018, Williamsburg, VA, USA

(C) 2018 Copyright held by the owner/author(s). Publication rights licensed to the Association for Computing Machinery.

ACM ISBN ISBN 978-1-4503-4911-6/18/03...\$15.00

https://doi.org/http://dx.doi.org/10.1145/3173162.3173213
Keywords Operating systems security, web application security, information flow control, information declassification

ACM Reference Format:

Casen Hunger, Lluís Vilanova, Charalampos Papamanthou, Yoav Etsion, and Mohit Tiwari. 2018. DATS - Data Containers for Web Applications. In Proceedings of 2018 Architectural Support for Programming Languages and Operating Systems (ASPLOS'18). ACM, New York, NY, USA, 15 pages. https://doi.org/http://dx.doi.org/10. $1145 / 3173162.3173213$

\section{Introduction}

Web applications have to implement a wide array of security features, such as input sanitization, authorization, and access control checks [13]. This burden often causes developers to implement these features incorrectly $[8,18]$ or fail to update vulnerable libraries promptly [19], making web applications particularly susceptible to "zero-day" vulnerabilities [5]. A compromised web application can then exfiltrate data to unauthorized users and cause large data breaches. Moreover, the threat of exploits forces considerable penetration-testing and compliance-certification work that slows down application development.

Ideally, users and enterprises would store their data on storage platforms (e.g., Google Drive or electronic medical record (EMR) systems), use untrusted web applications that integrate with these storage platforms, and still protect their data from being breached - i.e., enforcing mandatory access control (MAC) over the untrusted applications. Figure 1 shows a simplified setting where a doctor (Dave) shares folders with patients Alice, Bob, and Eve and uses untrusted applications for messaging and scheduling.

A natural defense strategy is to use data containers: run an entire application instance within the context of each data object, with each instance isolated into a separate container[35, $43,64,77]$. The access control rules will hold by definition even if an application instance is compromised or malicious (no information can be transferred across containers for different data objects). One could use language-level information flow control (IFC) to achieve the same goals (e.g., 

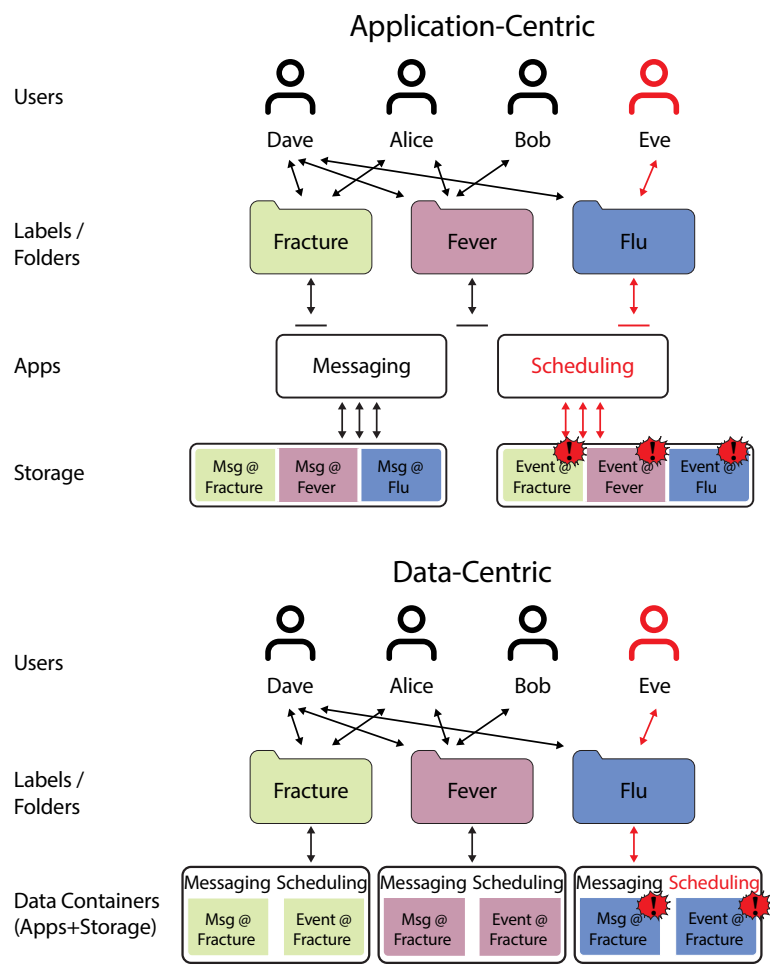

Figure 1. Current systems have application-centric access controls (top) and cannot prevent data leaks; e.g., a compromised or malicious "Scheduling" application can leak Alice's events to Eve (from "Fracture" to "Flu"). Data-centric access controls (bottom) enforce users' ACLs on all applications: data is confined to its respective label.

Hails [38] provides a Haskell framework that attaches labels to data in database models), but this compromises programmability. Developers rely on large bodies of existing frameworks and languages, therefore we cannot limit them to only using vetted options. The advantage of most OS container technologies is that they can isolate unmodified code.

Data containers raise two new challenges. Usability: for example, a calendar application cannot aggregate the information from appointments in different data objects into a single page. Efficiency: applications cannot use a single storage service, like deduplication, across data objects.

In this paper we present the DATS system, which refactors authentication and access controls outside of untrusted web applications. DATS couples data containers along with two new robust declassification [76] mechanisms in a novel way to trivially enforce access controls at the container level. It leverages the model-view-controller (MVC) pattern, common in many web applications, to provide application programmability by presenting a familiar programming model to developers and supporting several existing web application languages and frameworks.

First, DATS recovers usability by securely composing views from multiple data objects. Applications can provide an untrusted view template to a trusted template declassifier to aggregate information from each per-data object container into a single page, like in many templating languages [16]. The declassifier transparently applies language level IFC to prevent information leaks across data objects.

Second, DATS improves efficiency by securely sharing untrusted storage services across data objects. Applications can use a trusted storage declassifier that interposes between applications and untrusted shared models (e.g., for deduplication, key-values stores, compression, etc.). The declassifier performs integrity checks on each data read operation (i.e., "get $\left(\operatorname{key}_{x}\right)$ ") to ensure that it only returns the value from the most recent "put (key, val)" operation on the same data object - interestingly, we use this to ensure that storage does not leak information across data objects.

Container technologies are available in production systems, but replicating an application per data object has intrinsic inefficiencies known as multi-execution [26]. Performance is largely secondary for enterprises (they have far fewer users than internet-scale services), but multi-execution can become problematic when operating across a large number of data objects (e.g., search). Therefore, we explore using hardware-assisted thread containers to avoid multi-execution.

DATS and the client browser are the only components that reside in the trusted computing base (TCB), while ensuring applications remain programmable, usable, and efficient. IFC efforts like Hails [38] and Jeeves [74] are instead a great fit to the trusted developers of DATS's TCB. Enterprises can then leverage the vast space of existing and untrusted web applications, frameworks, languages, and developers while at the same time avoid costly application code audits. We make the following contributions:

- We introduce DATS, a programming model that enables web services to be run in data containers - DATS uses two new robust declassifiers to retain functionality and OS-level multi-execution to minimize constraints on programmers (§§ 3 and 4 ).

- We evaluate programmability and security by developing 4 applications and porting 4 existing ones ( $§ 55$ and 6).

- We evaluate performance with existing OS-level containers $[10,60]$ and a HW-capability architecture [32, 66] (§ 7).

\section{Motivation}

Many enterprises, like hospitals, use web-based applications for security-sensitive data. Currently, the TCB includes every application since an application-level exploit can put all data at risk. Such applications are thus built, certified, and audited for security first, with performance being a secondary concern [9]. This accrues large costs from highly skilled, security-aware programmers and requires arduous 
penetration-testing and compliance-certification work before the smallest changes can be pushed into production. This is clearly at odds with rapid application development and deployment cycles and cost-efficiency; one cannot employ the vast majority of existing web developers, who are not security-savvy, nor leverage existing applications and development frameworks. We therefore need a systematic approach to provide security and cost-efficiency for such security-sensitive applications.

Figure 1 shows a simplified setting where a doctor (Dave) shares folders (i.e., access control domains or security labels) with patients Alice, Bob, and Eve and uses untrusted applications like "Messaging" and "Scheduling". Access control lists (ACLs) provide users a simple and intuitive way to express data confidentiality expectations for many applications; e.g., Dave explicitly decides to share folder "Fracture" with Alice in Figure 1. Our "folders" are the "control domains" in security literature, and folder ACLs serve as security labels for the data inside folders.

Existing systems provide application-centric policies (top of Figure 1); they can isolate applications from each other, but a compromised application can access data from multiple access control domains, even if users correctly set their folders' ACLs. For example, a buggy or malicious "Scheduling" application can store events intended for folder "Fracture" into "Flu" when processing Dave's appointments. Even if all applications restrict Eve to only access "Flu", this folder now contains events exfiltrated from "Fracture". In the worst case, data from all folders could be exfiltrated, violating all user confidentiality expectations. Instead, a data-centric MAC policy (bottom of Figure 1) provides an intuitive alternative, where data from different folders cannot interfere with each other.

In this section, we describe the challenges in enforcing data-centric MAC over untrusted applications, and the opportunity inherent in the structure of MVC applications to refactor security out of the application requirements.

\subsection{Threat Model}

Our threat model for web applications has the client browser and the server-side platform (i.e., the hardware, hypervisor, OS, and DATS's core components) as part of the TCB. The applications, their libraries, and storage services (e.g., key-value stores) are hosted on the platform but are outside the TCB; this also includes application code running on the client's browser (e.g., using JavaScript). DATS aims to prevent the following attacks by refactoring authorization and access control out of the untrusted components:

Code-driven folder interference. Untrusted code and developers are considered an attacking subject - either directly through malicious intention or indirectly through buggy code - and must not be able to leak data across any two folders (or arbitrary internet addresses). In information flow terms, the user's requirement is non-interference [39] across folders.

User-driven folder interference. Users (authorized or not) are considered an attacking subject and must be prevented from violating non-interference across unauthorized folders. This includes exploiting untrusted code through memory errors, malicious inputs, etc. or running arbitrary code to access unauthorized folders.

We otherwise consider authorized users trusted; they fully delegate trust in handling sensitive data to other users with whom they share folders. We do not protect against poor judgment when sharing a sensitive file with another user, who is free to manually communicate its contents to another authorized folder or anywhere else.

We limit the scope of our problem and list complementary techniques to handle other risks to user data:

- Integrity: We do not prevent untrusted applications from mangling user data or destroying it. Systems such as Frientegrity [36] handle it orthogonally.

- Information leaks via timing or termination channels: We assume techniques that either normalize (e.g., deterministic execution [25] or predictive mitigation [23]) or randomize (e.g., fuzzing [65]) the timing of outputs (e.g., on a storage $\rightarrow$ app channel, possibly with behavioral/anomaly detection for termination channels).

- Privacy-preserving data mixing: Cross-folder functionality such as analytics (e.g., clustering or training classifiers) fundamentally violates the access control policies set by users. Complementary approaches such as differential privacy $[34,50,51,55,56]$ or quasi-identifier based privacy $[46,49,63]$ show that this functionality can directly integrate with DATS: the functions can be executed in isolation and only their perturbed output released (e.g., GUPT [51]). Similar declassifiers can be built for advertisement impressions [44] and for sending debugging output [27] back to developers.

\subsection{Challenges in Enforcing Data-Centric MAC}

OS-level MAC. The simplest way to enforce data-centric $M A C$ is to run an application process per folder inside an OS-level container using LinuX Containers [10], SELinux MAC [60], capabilities [69], or OS-level IFC (OS-IFC) [35, 43, $52,77]$.

This approach curtails crucial functionality. It cannot support web pages that display data from multiple folders (i.e., cross-folder views), since the application has limited access to a single folder. For example, Dave will need to open each per-folder "Scheduling" application to manually find a free slot for a meeting, a tedious and error-prone operation.

A per-folder application instance can also be inefficient, since a single MVC model cannot optimize storage across folders. For example, data deduplication has to work across all folders in order to find unencrypted data to consolidate. 
Storage services such as in-memory key-value stores (e.g., Redis, memcached) or distributed coding also work across folders and will be inefficient if we run a separate instance on each folder. Furthermore, running multiple instances of an application can sometimes lead to poor resource utilization in the micro-architecture (i.e., multi-execution [26]).

PL-level MAC. Programming-language (PL) level IFC [38, $58,75]$ can control the flow and aggregation of information inside an application (such as creating cross-folder views) and enforce data-centric MAC policies. However, it requires careful annotation of program inputs, intermediate variables, and outputs to ensure information does not flow between inputs and outputs with incompatible ACLs. This makes PL-MAC notoriously complex to use in practice, requires developers to have extensive security expertise, and constrains them to specific programming frameworks. Further, PL-MAC only works as long as application developers are trusted [58] or when the trusted platform developers maintain the data model for all third-party applications [38].

Finally, optimizations, such as deduplication, cannot be represented in a PL-MAC solution, since it compares data across folders and executes code based on this comparison.

\subsection{Opportunities in MVC Applications}

MVC frameworks are popular in web applications and require developers to write separate components and strict interfaces between views and controllers as well as controllers and models. We can use these interfaces to transparently enforce data-centric MAC over the entire web application.

Views in MVC applications separate presentation from application logic using templating languages. These languages are meant to arrange data in different ways instead of creating new information from data. This allows us to impose PL-level MAC without being undermined by pointers, reflection, and other features of full-featured languages.

Model (i.e., storage) optimizations implement diverse functionality in arbitrary languages, but they do not affect the ACLs of user data. Furthermore, several popular and complex web services expose variants of a simple put-get interface: deduplication, in-memory key-value stores (memcached), and cloud-based storage services such as Amazon S3 and Google Drive. Interposing a transparent integrity-checking proxy on this common interface enables untrusted services to work with plain-text data. This is essential for services like deduplication (that will not work if data is encrypted) and allows services to compress, index, or otherwise optimize storage across all folders.

\section{Design of the DATS System}

DATS ensures that untrusted applications cannot mix information across folders by executing a unique web-application instance for each folder. In this section, we describe how
DATS enables untrusted applications to implement crossfolder views and storage without breaking this folder noninterference policy. We also describe hardware-assisted OS containers that help reduce the overhead of multi-execution in DATS. We begin in Figure 2 with a typical user workflow and walk through the key steps in the lifecycle of an application on DATS.

A user begins by visiting the DATS Desktop (§ 3.2). Here, DATS exposes Data objects (called folders) to users, similar to platforms like Google Drive, Box, or Dropbox. Users express their confidentiality policies (i.e., who can access which folders) using simple per-folder ACLs. Every folder can hold arbitrary data, making them independent of applicationspecific constructs like messages, documents, medical records, etc. DATS enforces folder-level ACLs on untrusted thirdparty applications that attach to users' Drive/Box accounts. Folders are color-coded in the figure (green, red, and blue) to easily track information flow across the system. DATS implements users, folders, and folder ACLs. Thus, applications need not implement further ACL rules.

After managing their ACLs, a user selects an application and launches its landing page $A$. On this page, users can set the application's preferences used across all their folders, like profile pictures, since this data is made explicitly public to all folders. We term this as a non-folder view. From this page, a user can trigger a cross-folder view of information $\mathbb{B}$, like a list of upcoming appointments. The user can then click on a specific appointment to load its per-folder view $\mathbf{C}$ and get or set additional information. From here, a user can move between cross-folder (appointment list) and per-folder (appointment information) views.

We will now describe the server-side actions using the same workflow. First, DATS starts an application in a nonfolder container (1), which cannot access user data from any folder (only sees the user's public settings and application resources). DATS uses this container to create cross-folder views (2a and (2b leading to $\boldsymbol{B}$ ) and then sets up per-folder containers in 3 as the user traverses links out of cross-folder into per-folder views. We now describe the developer's view of application components on DATS.

App components contain entire (untrusted) applications and are instantiated multiple times. Each instance runs in an OS-level container $[4,10,59]$ (app row in Figure 2) with a trivial data-centric MAC policy: it has exclusive access to a single folder and no other external resources. Containers get a standard system view, including system calls, libraries, and runtimes for any development framework and also extend to the client browser. This is key for programmability given the diversity in toolkits and languages for developing applications.

This "data container" approach, however, forbids crossfolder functionality, breaking the usability and efficiency 


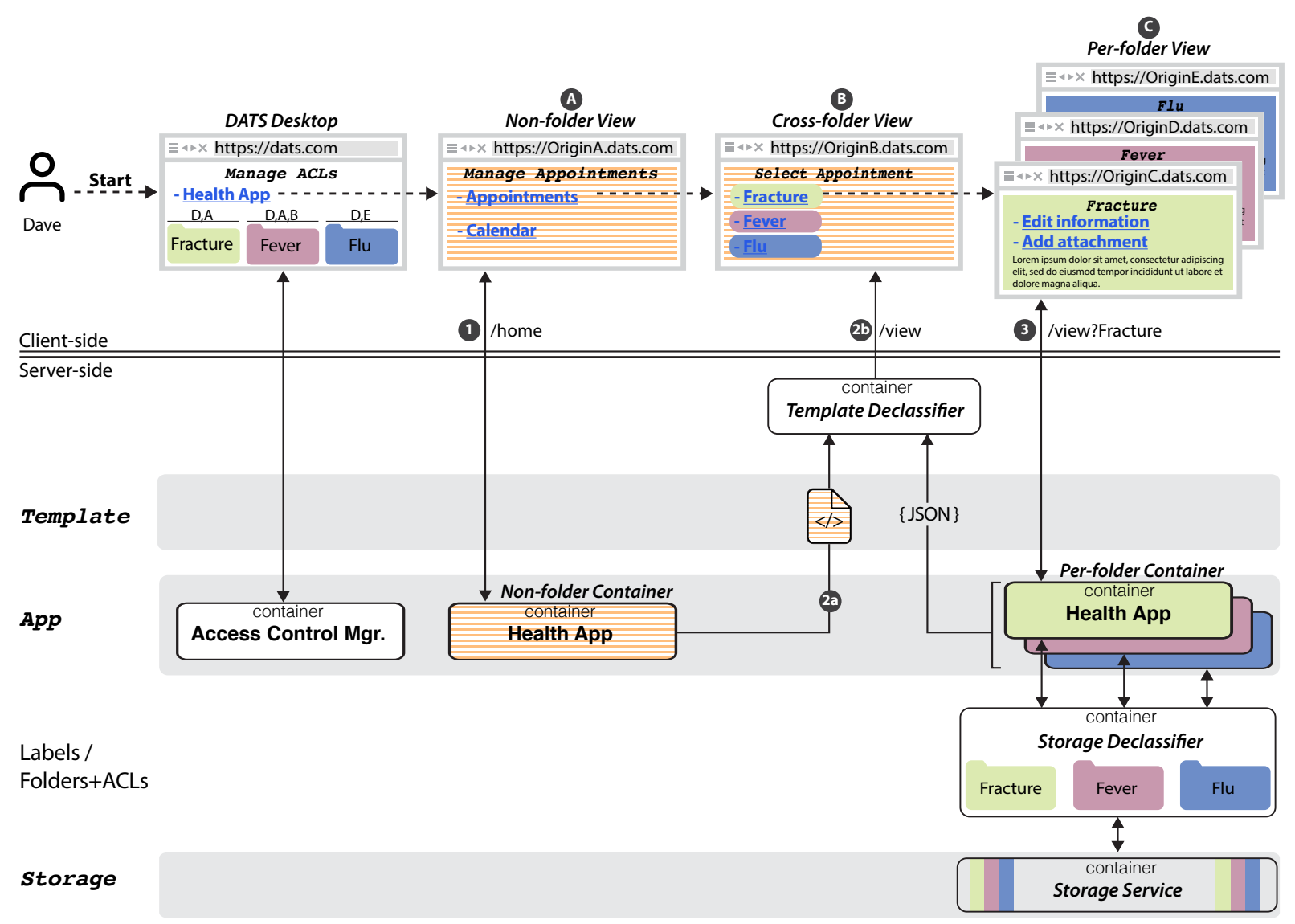

Figure 2. Example web page flow from a user (“client-side"), DATS's main components, and an application's app-template-storage components (and their relation to MVC). Application code, application data, and storage services are untrusted (grayed areas and colored boxes), while DATS's trusted components (boxes with white background) enforce folder non-interference. Application components run inside OS-level containers, which can very easily enforce per-folder MAC policies. Note that the client's browser is allowed to run untrusted application code (e.g., JavaScript).

of most applications. DATS thus provides two robust declassification [76] mechanisms for cross-folder functionality, ensuring that untrusted code cannot affect declassified data.

Template files describe how to aggregate information from multiple folders into a single cross-folder view, making applications usable. The template in Figure 2 is provided by the non-folder app in 2a. A trusted Template Declassifier inflates templates with data from each per-folder app instance (2b) through a simple form of PL-level IFC: it generates HTML/JS by processing only one data element at a time, and each output (e.g., link) can only send information back to the app instance that produced it (e.g., (3).

Storage services (untrusted storage row in Figure 2) implement the application's cross-folder models to make storage efficient. DATS interposes a trusted Storage Declassifier on the app-storage communication. The Storage Declassifier uses integrity checking to ensure that each response value of a "get (key)" request from a folder is the same as the last "put(key, value)" for that folder. Interestingly, integrity checking the put-get interface and confining the storage services in a container enables these untrusted services to work with plain-text data.

We will now discuss the security invariants in DATS (§ 3.1) followed by details about each component (§§ 3.2 to 3.6).

\subsection{Security Invariants in DATS}

DATS guarantees end-to-end folder non-interference, covering apps and information flow-secure views and storage. Each app instance runs in a confined container - conceptually, each container covers server and client devices - with access to only one folder. This ensures baseline folder noninterference for app instances.

DATS's Template Declassifier inflates untrusted templates from the application with untrusted results from many folders to construct a view, but ensures that communication from the view back to an app instance only uses data from that same instance. The Template Declassifier prevents explicit information flows by ensuring each HTML element that can send a request (e.g., a link) contains information from at most one folder and points to the app instance that 
has access to it. It also prevents implicit information flows by using a template language that does not allow predicates or conditional loops over results from different folders. Fortunately, view templating languages are already moving towards such restrictions to minimize business logic inside the presentation layer [12].

DATS's Storage Declassifier ensures that a get value returned to an app equals that last value that was put by the app - hence, the untrusted storage service can operate on plain-text data across folders and yet be prevented from breaking folder non-interference. Integrity checking in Storage Declassifier not only prevents storage from explicitly copying Alice's data into a get response for Bob, but also prevents implicit leaks where storage returns Bob's value $\mathrm{X}$ if Alice's secret bit is 0 and Bob's value $\mathrm{Y}$ if Alice's bit is 1. storage is confined to a container with no outputs other than to the Storage Declassifier, assuming that timing/termination channels from storage to app are addressed using complementary techniques (see § 2.1). DATS's Storage Declassifier and confinement together demonstrate a novel method of using an authenticated storage interface to enforce noninterference.

DATS's guarantees rely on a few fundamental primitives: containers on server and client, IFC in the Template Declassifier, and integrity checking in the Storage Declassifier. The Template Declassifier and Storage Declassifier provide robust declassification [76] for cross-folder functionality - i.e., guarantee that untrusted code or data in a folder cannot affect messages/data that is sent from a cross-folder container back to a different per-folder container.

DATS extends containers into the client browser by using one sub-domain per app instance (browser origin in Figure 2). DATS then activates the Same-Origin Policy (SOP) and Content Security Policy (CSP) on the client browser to limit access to remote resources and confine untrusted client-side code (e.g., JavaScript) within each sub-domain (container). DATS also drops all cookies set for parent domains. Containers on the server can be implemented directly as reference monitors (e.g., SELinux or LXC), with capabilities, or with information flow control - DATS deployers can pick one based on performance and compatibility constraints.

Components interact with DATS abstractions using an authenticated RESTful interface. Therefore, trusted components are no different than the untrusted ones, except that they are authorized to perform operations such as user authentication. Internally, each container is identified by the application it is running (e.g., Health), the user it is running for (e.g., Dave), and the folder it has access to (e.g., "Fracture"). As used throughout this section, the green container in Figure 2 would be identified as $<$ Health, Dave, Fracture $>$.

\subsection{Data Object (folder) and User Management}

DATS provides users with two trusted user interface (TUI) applications - Login authenticates and manages users, and the Desktop allows users to create, delete, and share folders and launch untrusted applications. Access control over folders lets users control the granularity at which their data is shared. A user may wish to assign a single medical encounter to a folder, allowing them to share each of their encounters individually, or a user may wish to place all their encounters within a single folder, allowing them to share their entire medical history at once. While users determine how much is shared, developers control the minimum unit of sharing.

DATS provides two services to retain application functionality after removing access-control and authentication: app instances contact the User Service to see which users have access to their current folder, and the Template Declassifier uses templates to create cross-folder views (§ 3.4).

DATS provides the User Service because applications work with the 'user' abstraction for a large portion of their functionality, in addition to access control, and removing the concept of users from applications only works for the simplest of applications. For instance, Mattermost (see § 5.1) has 92 different SQL queries involving its user table, some of which are complex and include joins across multiple different tables. The User Service eases application development by allowing per-folder app instances to read information about the users who have access to the apps' folder and to trigger notifications to other app instances associated with the same folder (e.g., those running for another user).

\subsection{App Components}

The app components contain most of the application logic and are run within containers. They communicate with client browsers through the trusted DATS Proxy (not shown in Figure 2 for clarity). The Proxy routes traffic between the sub-domains used by client browsers and the app instances (i.e., containers) on the server. Non-folder and per-folder functionality are the most common (1) and 3) and can have native performance (DATS acts as a reverse proxy between the client browser and app instances).

The Proxy extends containers to the client side to enforce indirect folder non-interference: it activates the browser's SOP and CSP and drops cookies for parent domains (see § 3.1). Every time an app is instantiated it is assigned a new random sub-domain. For example, DATS maps the non-folder view in A of Figure 2 to the app instance $<$ Health, Dave $>$ (1), without access to any folder), and the per-folder view in C to the instance $<$ Health, Dave, Fracture $>$ (3), with access to folder "Fracture"); the cross-folder view in B also has its own sub-domain, but its contents are served directly by the Proxy (see § 3.4).

DATS has a trusted Container Manager that can be instantiated in multiple nodes to scale horizontally. It enforces direct folder non-interference by creating mutually isolated containers: each container is assigned an IP and port to listen for requests and only has access to its assigned folder (if any, since non-folder app instances cannot access any folder) and 


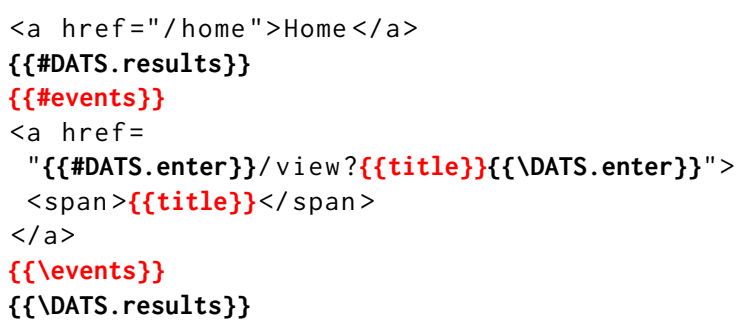

(a) Template

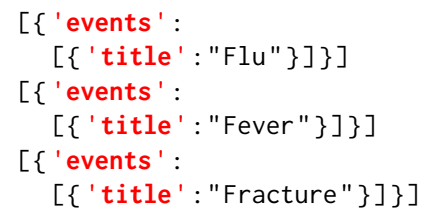

(b) Per-folder JSON data

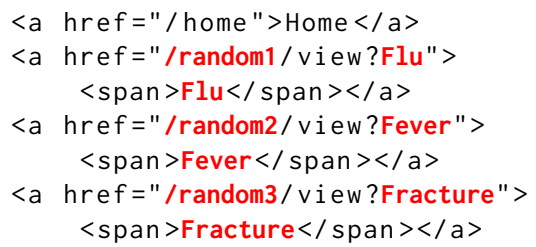

(c) Expansion

Figure 3. A cross-folder template and an example expansion from the DATS Health application. DATS keywords are black and bold and DATS Health's per-folder data is highlighted in red. Some of the HTML has been omitted for brevity.

to DATS's public API (see § 3.6). To avoid the latency costs of spinning up containers on-demand, the Container Manager uses container reuse, pooling, and prefetching.

\subsubsection{Hardware-Accelerated Thread Containers}

By default, DATS uses OS-level containers [4, 10, 59] to enforce isolation (such as LXC and SELinux as described in $§ 4.1$ ) and make applications programmable (developers can pick from many application stacks in existence today). Nonetheless, using multiple app instances has inherent multiexecution overheads [26], which are especially acute when a cross-folder view, such as search, requires streaming through all folders that a user has access to. Clearly, such large sweeping operations will put container startup and network setup latencies in the critical path of a view query.

To address this slowdown, we have designed thread-level containers in DATS. We use SELinux [60] and fine-grained capability-based architectures [32, 45] to build "thread containers" so that the DATS Proxy and app code can execute in the same process. This allows the Proxy to trigger a large number of thread containers - one app runs in each thread and performs the query (e.g. search) over data in one folder. $\S \S 4.1$ and 7.2 describe our implementation of hardwareassisted thread containers, even though one could also use a DIFC architecture like Raksha [31] or Loki [78] instead (where each folder would be a mutually unordered label in the policy lattice). Interestingly, thread containers are in line with event-triggered plugins used on a web server frontend (e.g., WSGI [20]).

\subsection{Template Components}

Cross-folder views retain critical functionality to make applications usable, but must maintain folder non-interference. Templates compose views declaratively with information from multiple folders without executing any untrusted code. Developers can use any static resource in cross-folder views along with some trusted JavaScript provided by DATS.

Templates are written in a simple language, like views in many existing MVC web applications [16]. They are based on the stateless Mustache language [12] (without explicit control-flow operations like loops), making it easy to apply IFC to ensure folder non-interference ${ }^{1}$ : each element in B contains information from at most one folder and can only send a request to the app instance that provided that information (e.g., URLs for images, links and forms).

The Template Declassifier forbids arbitrary JavaScript in templates, since current browsers do not provide IFC on client-side code (e.g., unlike COWL [61]). Instead, the Template Declassifier recognizes additional tags that expand to trusted snippets of JavaScript for searching, sorting and autocompleting elements (see Table 1). Such tags can also be used to generate scripts that integrate differential privacy databases like PINQ [50] into a DATS application.

In practice, we found that a most application functionality lies in non-folder and per-folder views (and can therefore include arbitrary JavaScript). Also, programmers can use their own templating language to produce a valid template.

\subsubsection{Cross-Folder View Example}

This section explains the steps involved in using a crossfolder view for the examples in Figures 2 and 3.

First, the user clicks the "Appointments" link A in their browser and an HTTPS request for /view is sent to the Proxy. The Proxy determines the app instance to forward the request to based on the request's sub-domain. The nonfolder application responds with a template 2a (Figure 3a) and triggers a view of data across all folders by setting the $\mathrm{x}$-dats-crossfolder header field.

The Proxy redirects the client to a new temporary subdomain B while it constructs the cross-folder view. The Proxy creates (or reuses) an app instance for each of a user's folders and replays the /view request to each of them. Each per-folder app instance responds to the request with a JSON list (Figure 3b). The DATS API informs App instances if they

\footnotetext{
${ }^{1}$ The same approach could also be applied to any application generating user-facing views (e.g., using Android layout templates). A purposely simple templating language side-steps the precision vs. soundness problems of applying IFC to full-featured languages $[58,68]$.
} 


\begin{tabular}{l|l}
\hline Endpoint & Usage \\
\hline $\begin{array}{l}\text { User Service } \\
\text { /users } \\
\text { Proxy } \\
\text { /port }\end{array}$ & Get per-folder user info. \\
Application & Connect to a storage service. \\
/dats/start & \\
/dats/update & Send folder and user info to apps. \\
/dats/quit & Send updated user info to apps. \\
Template & Apps return from per-folder view. \\
DATS.results & Inflate region with per-folder info. \\
DATS.enter & Create per-folder URL. \\
DATS. search & Trusted JS for searching tags. \\
DATS. sort & Trusted JS for sorting by tags. \\
DATS. autocomplete & Trusted JS for autocomplete by \\
& tags. \\
\hline
\end{tabular}

Table 1. Public API in DATS. User Service, Proxy, and Template endpoints are implemented by DATS. Application endpoints are implemented by untrusted apps.

are executing in non-folder or per-folder mode so that logic on the "shared" / view endpoint can change accordingly.

The Template Declassifier collects the per-folder results and uses them to inflate the template (2b). The template's top-level tag, DATS. results (Figure 3a; there can be many, but not nested), is inflated with each per-folder result in turn (Figure 3b). The result is served by the Proxy as the cross-folder view in B.

URLs in inflated regions need to use the DATS. enter tag or an error is returned. The tag adds a random prefix (tracked by the Proxy; see below) to URLs. Programmers can use their own tags to reference per-folder information inside a DATS. results block (e.g., red tags for events and title in Figures $3 \mathrm{a}$ and $3 \mathrm{~b}$ ).

The Proxy redirects a request for /home in Figure 3c (or any other resource outside the DATS. results region; outer orange region with horizontal stripes in (B) to the sub-domain for the non-folder app instance (i.e., that serving (A)). A request for /random3/view?Fracture (or any resource created with DATS. enter; inner folder-colored regions in $B$ ) is instead redirected to the sub-domain for the corresponding per-folder app instance (i.e., that serving $\mathbf{C}$, by stripping the random3 prefix to get the endpoint / view?Fracture that 3 will serve).

\subsubsection{Non-Secure Alternatives}

The intuitive solution to creating cross-folder views based on OS-level IFC would be to create an app instance with read-only access to all folders. This folder will acquire the highest secrecy label and cannot let users click on links in views (e.g., an inbox) to go to a message in a folder. This is because the app could embed information from folder "Fracture" into a message to a container for folder "Fever" (e.g., to edit an appointment there) and violate folder non-interference.

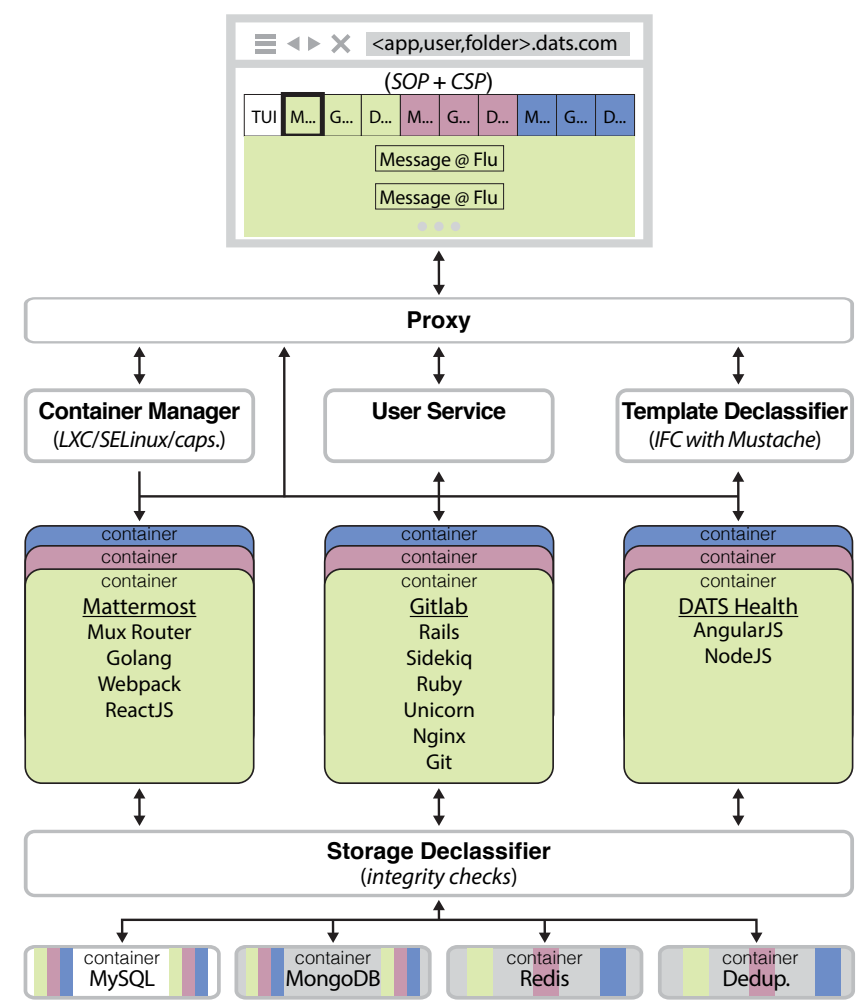

Figure 4. Implementation of DATS; colors and gray denote untrusted code and italics show the mechanisms used. All DATS components run in containers. The TUI application is not shown for brevity.

Alternatively, one could whitelist specific URLs and the data sent through each URL to prevent explicit leaks as above. In this case, a malicious app can create an implicit leak by picking white-listed message A vs. message B to indicate 0 vs. 1 - the view component in DATS fundamentally requires information flow control inside a container. We emphasize that DATS could have used any language with information flow control for templates - we picked a logicless templating language since software engineering practices simplify the Template Declassifier's task.

\subsection{Storage Services}

Storage services increase application efficiency by running untrusted functionality on plain-text across folders (i.e., crossfolder storage services or models in an MVC application). Efficiency can be in terms of storage space (e.g., cross-folder data deduplication) or other resources (e.g., using a single service instance to avoid multi-execution [26]).

DATS isolates storage services inside a container, and app instances must use the Proxy's /port endpoint to request access to storage instances. It returns a connection to a transparent trusted Storage Declassifier (also inside a container) interposed to the storage service to ensure that it cannot take data from one folder and output it to another folder's app component. Storage Declassifiers associate the requesting app's IP to their assigned folder. 
Many popular applications have models using (variants of) put-get interfaces, where integrity checks are sufficient to ensure non-interference. The declassifier checks that a "get(key)" request returns the most recent value for "put(key, value)" (otherwise aborts the connection). The same approach can be transparently applied to other interfaces, like disk blocks or file systems [62].

Finally, many large code-bases use trusted databases that have already gone through extensive audits (e.g., trusted developers assign roles to implement access policies). In this case, the declassifier creates per-folder databases on a single storage instance and uses the existing access control mechanisms to limit each app instance to its corresponding folder's data. The database should maintain data integrity, but that is not a requirement (e.g., IntegriDB [79]).

\subsection{Application Instance Lifecycle and APIs}

Table 1 summarizes the API available to untrusted components whose lifecycle is managed by the Proxy. Even if not shown in the figures for clarity, all containers have read-only access to a folder that contains their code. Non-folder containers also have read-write access to a folder that can be used to store user-specific settings, and per-folder containers get read-only access to it (ensuring folder non-interference). Perfolder instances cannot directly link back to a non-folder or cross-folder view since that could be used to exfiltrate information across folders. Instead, the Proxy intercepts requests to /dats/quit and redirects the client to the cross-folder view that initiated a transition to that per-folder view.

\section{Implementation of the DATS System}

Figure 4 shows all the components described in $\S 3$ and how they interact, with an emphasis on DATS's TCB. It includes four Storage Declassifiers we wrote for MySQL, MongoDB, Redis, and a custom deduplication backend. We wrote $13 \mathrm{~K}$ lines of TCB code in Python, Node.js, and C. The TCB can be substantially reduced by running containers on securityoriented OSs like seL4 [42] or HiStar [77]. Instead of trusting the hypervisor, we could also use attestation and trusted boot $[41,54]$ to bootstrap DATS on a remote cloud.

MongoDB and Redis are not limited to put/get, but we find this sufficient for our diverse applications (see § 5). Also, app programmers can always use a per-folder instance of their storage service.

The Proxy is a multi-process Flask [6] application running behind Apache with MySQL as a persistent database, Redis [15] as a short-term database and for distributed locks, and Celery [2] to run cross-folder requests in parallel. API operations are authorized by inspecting the requestor's IP; this is the simplest mechanism that can monitor folder access grants (each container has a unique IP).

The Proxy authorizes access to container sub-domains using a session cookie set by the trusted Login application and also performs a simple form of caching of the per-folder JSON results used to feed templates. The caching API is not described due to space constraints, but follows concepts similar to existing web caching technologies.

\subsection{Container Backends}

Off-the-Shelf Container Techniques. LinuX Containers (LXC) [10] provide lightweight OS virtualization; we did not try conventional VMs since LXC is more efficient [37, 57]. Containers are created as a "clone" of a base file-system with additional folders overlaid on top using AUFS [1] and are isolated at the network level using iptables.

SELinux $[48,60]$ implements flexible and fine-grained MAC for Linux. Each container gets a virtual network interface and a binary policy module with the necessary labels to access files and network ports.

HW-Capabilities Based Thread Containers. When a Proxy thread "enters" a container (executes a per-folder request using the app code), we prevent it from sharing memory with other threads using the CODOMs capability architecture [66]. The app code is loaded into a separate CODOMs protection domain that isolated threads have read and execute access to. Each thread gets a read-only capability pointing to its input request and two read-write capabilities that point to their private stack and heap pool, respectively. To prevent sharing through the file system, we added a Linux system call (used by the Proxy) that privatizes the thread's file descriptor table and SELinux label. When a thread returns from its app call, the Proxy restores the per-process file descriptor table and SELinux tag, and frees the thread's private memory pages (the private heap and stack).

\subsection{Enforcing Client-Side Non-Interference}

DATS uses the Template Declassifier and standardized browser security controls (configured by the Proxy on the headers of the responses it serves to the client) to avoid:

Leaks through request URLs/contents. The trusted Template Declassifier knows the HTML structure and semantics of tags that trigger browser requests (e.g., links and forms) and include untrusted client-side code (forbidden in cross-folder views). Each tag includes information from a single DATS. results block, ensuring that request URLs and contents have information from a single folder (i.e., a $<$ form $>$ block cannot span across multiple DATS. results blocks). Also, all URLs inside a DATS. results block are generated with a DATS. enter block - making sure the URLs cannot leak per-folder data through a request to another container.

DATS sets the browser's CSP to only allow trusted clientside code in cross-folder views (otherwise untrusted code could observe and modify a view to leak information).

Leaks through request headers. DATS's Proxy drops cookies set by untrusted app responses for any origin not 
assigned to that app instance/container (i.e., drops cookies for parent domains). The browser's CSP (and the newer "Referrer-Policy") ensures no data is leaked through the "Referrer" header (i.e., each view type is on a different origin).

Leaks to third-party domains. The browser's SOP forbids client-side code to access other non-origin domains.

Leaks through URL guessing. Randomizing container sub-domains ensures apps cannot exfiltrate information by generating a link or redirecting to an arbitrary sub-domain.

\section{Programmability Evaluation}

Developers can write or port applications to DATS without any security expertise, since authentication and access control are offloaded to DATS. Instead, they need only to make functionality-based decisions: establishing a minimum unit of sharing (§ 3.2), writing templates for cross-folder views (§ 3.4), and deciding what storage services to use (if any; $\S 3.5)$. We evaluate programmability in DATS by porting 4 open-source applications and writing 4 applications from scratch, all summarized in Table 2. They cover diverse usecases, like messaging, voice/video chats, document editing, software development, and electronic medical records.

We describe Mattermost and DATS Health in detail since they stress user management and template creation tasks.

\subsection{Mattermost}

Mattermost (version 3.7) is a messaging application written using Go (server side), React [14] (client side), and MySQL for storage. It has the concepts of teams (user groups) and channels to organize conversations based on similar topics.

We selected channels as the minimum unit of sharing, since they are a natural choice. A user can thus choose to create multiple teams and channels on a single folder or use only one channel per folder from the DATS TUI. The developer only provides the functionality of channels and teams as a way to organize data without tying it to access control (see § 3.2).

\subsubsection{Cross-Folder Views}

Mattermost by default lacks the ability view a single unified "inbox" of messages across all teams (the user has to enter a team and channel to view messages). We wrote a new landing page with a template (140 lines of HTML, application tags, and DATS tags) that displays a timeline of recent activity across all teams. The template includes application-specific tags (e.g., Teams) embedded within the DATS. results block. The per-folder request returns a JSON list with the teams, channels, and most recent messages for each channel stored on that folder. The template also uses the DATS. enter tag on each message to link it to a per-folder app instance that will show the full conversation.

\subsubsection{User Authentication and Access Control}

We removed user authentication from Mattermost and instead use the User Service to have an up-to-date per-folder

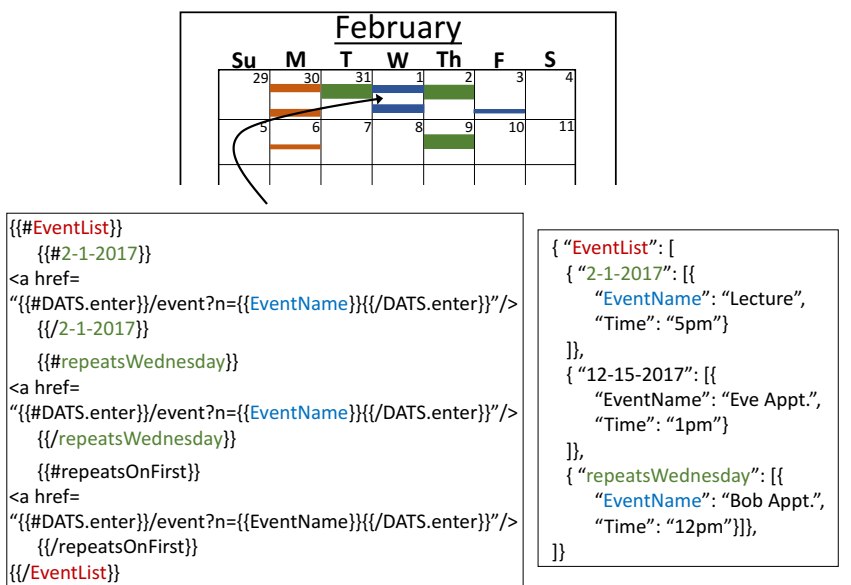

Figure 5. Snippet from the DATS Health calendar template page. Template tags (left) are matched with the per-folder FSON (right) and inflated to create the calendar information.

"Users" table (we added endpoints /dats/start and /dats/update; see § 3.6). This is crucial to Mattermost: e.g., it displays a list of recent activity for a particular team by issuing an SQL join query with its "Users", "TeamMembers", and "Status" tables.

\subsection{DATS Health}

DATS Health is an MVC application written from scratch by undergraduate students. It allows doctors and patients to manage medical records (each medical visit is an "encounter") and allows users to schedule appointments through three different calendar views (month, week, and day). Encounters were selected as the minimum unit of sharing. Unlike Mattermost, calendar views in DATS Health require an extensive use of templates (endpoints /month? <value $>$, /week?<value $>$ and /day?<value $>$ ).

Figure 5 shows an example of how we constructed the (non-folder) template and the (per-folder) JSON results for the /month endpoint (the other two work in a similar way). The template contains an empty grid with non-event information, such as month name and dates for each cell. Each cell is a DATS. results block contains application-defined tags that match the corresponding JSON results.

The JSON results are pulled from a MongoDB database and returned when a per-folder app instance receives a request to the same /month endpoint. Each event is embedded in the EventList array in one of three types of tags. The first one is a list of all events for the actual date (e.g., 2-1-2017). The other two tags are for weekly (e.g., repeatsMonday, repeatsTuesday) and monthly events (e.g., repeatsOnTheFirst, repeatsOnTheSecond).

The Template Declassifier goes through each DATS. results block and iterates across each per-folder result (EventList). Any event with a matching template tag (2-1-2017, repeatsWednesday, and repeatsOnTheFirst in Figure 5) is then inserted into the template. 


\begin{tabular}{|c|c|c|c|c|c|c|}
\hline Application & Framework & $\begin{array}{l}\text { DATS Effort } \\
\text { (LOC) }\end{array}$ & $\begin{array}{l}\text { Total TCB } \\
(\mathbf{k L O C})\end{array}$ & CVEs & $\begin{array}{r}\text { CVEs } \\
\text { w/ Leak }\end{array}$ & Description \\
\hline Gitlab & Rails & $\begin{array}{r}+386-50 \\
A:+36 / T:+100 / P:+250\end{array}$ & $145.2 / 1,352$ & $13 / 83$ & $9 / 45$ & $\begin{array}{c}\text { Repository management } \\
\text { [Ported] Minimum unit: project }\end{array}$ \\
\hline Mattermost & Mux & $\begin{array}{r}+443-800 \\
A:+25 / T:+100 / P:+318\end{array}$ & $191.4 / 1,219$ & $42 / 55$ & $19 / 28$ & $\begin{array}{c}\text { IRC chat } \\
\text { [Ported] Minimum unit: channel }\end{array}$ \\
\hline Hacker Slides* & Flask & $\begin{array}{r}+150-100 \\
A:+13 / T:+124 / P:+23 \\
\end{array}$ & $16.9 / 1,482$ & $-/ 40$ & $-/ 19$ & $\begin{array}{l}\text { Slide presentation and editing } \\
\text { [Ported] Minimum unit: presentation }\end{array}$ \\
\hline Let's chat* & MEAN & $\begin{array}{r}+260-500 \\
A:+21 / T:+100 / P:+139 \\
\end{array}$ & $23.5 / 1,891$ & $-/ 25$ & $-/ 4$ & $\begin{array}{l}\text { Real-time messaging using websockets [17] } \\
\text { [Ported] Minimum unit: channel }\end{array}$ \\
\hline DATS Health* & MEAN & $\begin{array}{r}+101 \\
A:+36 / T:+30 / P:+35\end{array}$ & $5.6 / 1,284$ & $-/ 25$ & $-/ 4$ & $\begin{array}{l}\text { Doctor-patient appt. management w/ calendar } \\
\text { [New] Minimum unit: appointment }\end{array}$ \\
\hline DATS Coding* & Django & $\begin{array}{r}+130 \\
A:+15 / T:+100 / P:+15\end{array}$ & $1.2 / 1,545$ & $-/ 254$ & $-/ 169$ & $\begin{array}{l}\text { IDE for code editing with terminal } \\
\text { [New] Minimum unit: code files }\end{array}$ \\
\hline DATS PDF* & Node.js & $A:+15 / T:+25 / P:+50$ & $1.1 / 1,071$ & $-/ 25$ & $-/ 4$ & $\begin{array}{c}\text { PDF document viewer } \\
\text { [New] Minimum unit: PDF document }\end{array}$ \\
\hline DATS Image* & MEAN & $\begin{array}{r}+90 \\
A:+10 / T:+50 / P:+30\end{array}$ & $1.1 / 1,131$ & $-/ 25$ & $-/ 4$ & $\begin{array}{l}\text { Upload and display images } \\
\text { [New] Minimum unit: image file }\end{array}$ \\
\hline
\end{tabular}

Table 2. DATS Effort: lines of code (LOC) to make an application DATS-aware. Total TCB: LOC of just application code (left) and including all its dependencies (right). CVEs: public CVEs since 2013 [3] for the application (left) and entire application stack (right). CVE w/ Leak: CVEs which contain information disclosures for the application (left) and entire stack (right). With DATS, the entire application SW stack is outside the TCB and folder interference is systematically eliminated.

$\left(^{*}\right)$ : CVE information not available. (A): Application code. (T): template contents. (P): Use of DATS's APIs.

The repeats. . . tags are crucial to avoid producing large JSON results where an event must contain a key for every date it should be displayed on (up to 365 entries per event per year). Additionally, they allow for JSON result caching (see $\S 4$ ) across different templates.

\section{Security Evaluation}

DATS mitigates all data-disclosure vulnerabilities by refactoring authorization and access control out of untrusted web applications (i.e., moving applications outside the TCB). Table 2 summarizes the total number of vulnerabilities found in the applications and their third-party dependencies that we have run on DATS. Unprevented vulnerabilities include broken functionality that does not produce information disclosures, such as unauthenticated team creation in Mattermost ${ }^{2}$. We will now discuss vulnerabilities found in Mattermost and Gitlab to understand their root causes and how DATS prevents them.

Mattermost has disclosed 42 vulnerabilities since 2015 [11], 19 of which DATS would prevent. They include cross-site scripting, remote code execution, denial-of-service, message spoofing, and authentication bypassing. The majority stem from missed access-control checks and incorrect input sanitation. For example, a missed authorization check in /api/v1/users/find_teams allowed attackers to view the

\footnotetext{
${ }^{2}$ The data we collected for this evaluation can be found in https://bitbucket. org/datsplatform/security-evaluation.
}

invite link for any team on the system, provided that they knew the email of any user on the team.

We also examined the 13 CVEs ( 9 preventable by DATS) disclosed for Gitlab and discovered similar vulnerabilities. For example, Gitlab improperly sanitizes public keys uploaded by users before operating with them, allowing attackers to execute arbitrary code on a Gitlab server [7].

DATS prevents these improper data disclosures because each application instance is confined to its own folder, and user authorization is centralized in the TCB. Vulnerabilities can appear in application code, but also in the large stacks of third-party code used by web applications. In total, 402 CVEs have been reported for the stacks of the applications found here, of which 237 would be prevented by DATS.

Interestingly, DATS is also able to contain app-layer denial of service (DOS) attacks; we can apply per-container resource limits (e.g., using cgroups in Linux) to contain attacks to a per-user and folder instance. For example, incorrect sanitization in large file uploads allowed for a server-side DOS attack in Mattermost, consuming too much memory within the application. Similar attacks can also be seen in the CVEs for the applications and stacks in Table 2.

\section{Performance Evaluation}

We built DATS using two container technologies and show that HW-assisted containers improve performance. We find that DATS provides reasonable performance for the majority of application operations (per-folder requests) and good 
scalability for cross-folder operations (albeit with a fixed latency cost due to multi-execution § 7.1.2). For worst case operations (searching across all folders), a naive implementation introduces overheads of $70 \times(13$ secs) while hardware assisted solutions brings this down to $1.47 \times$ (47 msecs).

All experiments use an Intel Core i7-4770 (4 cores 2-way SMT @ 3.40GHz), 12 GB DDR3, an Intel 82574L NIC (1 Gbit), a Seagate ST3500413AS disk (500 GB, 7200 rpm, 16 MB cache) and Ubuntu server 14.04 LTS.

\subsection{Existing Container Technologies}

Container latency is encountered when creating containers, starting applications inside containers, and destroying containers. Starting an LXC or SELinux container along with the application inside ranges from about 1second (e.g., DATS Health) to tens of seconds (e.g., Mattermost). The Container Manager maintains a pool of free containers and prefetches them, hence none of these operations are on the critical path.

\subsubsection{Per-Folder Throughput and Latency}

We measured the average throughput and latency for a perfolder request to DATS Health (§5.2) with varying number of clients - using a low-overhead query to access a single encounter to focus on DATS's overheads. The baseline application (one process per client) is compared against a version running in DATS using either the LXC or SELinux backends, both with and without a shared MongoDB storage service.

We find that all experiments have a pareto-optimal point at 8 clients, with latency overhead of $3 \times(10 \mathrm{msec})$ and throughput overhead of $66 \%$ (1000 req/sec). DATS's scaling is limited due to its Proxy component since it routes all requests to app containers - a dynamic reverse proxy (for client authentication and routing to the target container) would greatly improve latency, contacting the Proxy only when a crossfolder operation is triggered. Throughput would improve by moving reference counting (now serialized in Redis) to a distributed algorithm with batched lazy releases. Latency and throughput would also improve with simple page caching.

\subsubsection{Cross-Folder Throughput and Latency}

We also measured the latency of a worst-case cross-folder request with an increasing number of folders (same configurations and disabling the JSON result caching from $\S 4$ ).

DATS shows a base overhead of $30 \times$ (from 0.01 to $0.3 \mathrm{sec} \mathrm{la-}$ tency) due to container-agnostic factors: (1) the latency overheads in $\S 7.1 .1$; (2) the additional redirects for cross-folder views (§ 3.4); and (3) a sub-optimal Template Declassifier using Python libraries. The folder count has a small linear increase in latency overheads (up to $1 \mathrm{sec}$ for 50 folders). This is inherent to the multi-execution of existing containers (each per-folder request needs a separate process), and storage consolidation shows a slight improvement on that.

DATS thus has reasonable folder scalability - container reuse and prefetching and running per-folder requests in parallel effectively hides costs. The base overhead can be

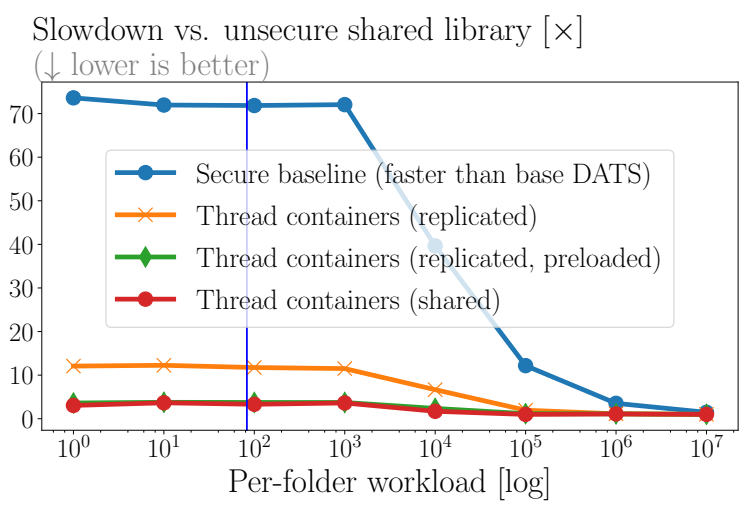

Figure 6. Performance of a cross-folder operation with hardwareaccelerated thread containers, normalized to the performance of an unsecure shared library. The Y-axis shows the normalized number of non-isolated threads while the X-axis shows the processing work on each folder. The unsecure baseline goes from 47 to $13,721 \mathrm{msec}$, and generating a cross-folder result for $\$$ 7.1.2 corresponds to the thin vertical line.

eliminated using known optimizations (§ 7.1.1) and a faster Template Declassifier implementation.

\subsubsection{Cross-Folder Storage Declassifier}

We also analyzed the latency overheads of using a Storage Declassifier with our deduplication storage (§ 4). The declassifier adds a $2 \times$ and $3 \times$ latency overhead for get and put operations, respectively. This is expected given our simple prototype (150 LOC of Python), but shows ease of implementation. More efficiency is possible through data blocking and using Merkle trees [62] for integrity checking.

\subsection{Hardware-Accelerated Containers}

Cross-folder operations are critical in DATS and existing container technologies bring intrinsic overheads: the multiexecution of per-folder processes [26] and copying data across them. We find that hardware-accelerated thread containers ( $\S 3.3$ and 4.1) help eliminate these overheads while even existing containerization technologies have negligible overheads for large application workloads.

Figure 6 shows the performance of a cross-folder operation with 800 folders using 8 threads.The $\mathrm{X}$-axis shows the workload necessary to make overheads negligible - each request calculates the factorial of the $\mathrm{X}$-axis number (stored in a per-folder file, opened and closed on each request). The Y-axis is normalized to the regular non-isolated threads. We ran the experiments natively, using the same methodology of previous studies with CODOMs [67 $]^{3}$.

Secure baseline shows the most efficient existing container technology with perfect Proxy scalability and SELinux label prefetching, where app and Proxy communicate through a pipe. Slowdowns range from more than $70 x$ to $1.47 x$. Ported

\footnotetext{
${ }^{3}$ Capability operations are emulated using regular memory accesses, and hardware registers are emulated using thread-local variables.
} 
applications see the highest overhead; the workload in $\S 7.1 .2$ corresponds to the vertical line in Figure 6.

Thread container (replicated) shows the costs of running a thread container with a regular app instance; the Proxy uses dlopen/dlclose when entering/exiting the container, respectively. The slowdowns are drastically reduced to $12.25 \mathrm{x}-1.01 \mathrm{x}$ because of less expensive thread containers and by avoiding copies between the Proxy and the app.

Thread container (replicated, preloaded) decouples the multi-execution overheads from library management by optimistically preloading all app instances and never unloading them. Slowdowns are $3.76 \mathrm{x}-1.01 \mathrm{x}$, since multi-execution still affects performance; having multiple virtual copies of a library does not allow optimal sharing of read-only and micro-architectural state.

Thread container (shared) shows the best results by sharing a single read-only app instance across thread containers. It removes the overheads of multi-execution and managing per-container library instances, reducing slowdowns to $3.65 \mathrm{x}-0.98 \mathrm{x}$, but global writable data must be replaced with dynamic allocations.

Thus, hardware-acceleration reduces overheads from $70 \mathrm{x}$ to $3.65 \mathrm{x}$ (worst) to within measurement noise (best).

\section{Related Work}

Hardware-assisted reference monitors (e.g., Intel MPX, SGX) $[22,40]$, capability-based [42, 66, 69, 70], information flow tracking [31, 43, 77], and other systems [73] can be used to implement containers. On the client side, data-confined sandboxes and IFC $[21,61]$ can improve DATS's security guarantees and template flexibility. Verifiable SQL queries such as IntegriDB [79] can be integrated as Storage Declassifiers, moving SQL DBs outside the TCB.

PL-level IFC solutions (Jeeves [74], Fabric [47], Aeolus [30], Jif [58]) allow for dynamic, fine-grained IFC abilities, but often require developers to understand information flow policies or limit choices to supported languages. These solutions are good candidates for writing DATS trusted components.

Other solutions segment data and applications along the user axis. Radiatus [29] runs applications inside user containers - collaborative containers will require DATS-like templates and a Storage Declassifier. Radiatus's capability protocol requires applications to be ported to access per-user data, similar to our API. It also trusts third-party storage services to maintain data separation between per-user privacy domains. Similarly, CLAMP [53] replicates a WebStack per-user with two security-critical pieces: a query restrictor (which guards a database) and a dispatcher (which authenticates the user). CLAMP does not enable controlled sharing of user data with untrusted code. $\pi$ Box [44] containerizes peruser applications and uses a differential privacy declassifier for ad-impressions.
Lastly, similar solutions provide segmentation at the dataobject level. Hails [38] lets developers associate access control policies to the model, while replicating view and controller components for each security label. Applications are written in Haskell and developers have to label their models (or platform administrators have to understand each application's models), and cross-label (i.e., cross-folder) views or storage services like deduplication will require extending Hails with our robust declassifiers.

Earp [72] allows users to share app-specific data objects (e.g., a custom animation inside an album) but requires developers to implement schema-level permissions (like Hails' MPVC framework). DATS's model is more appropriate when users wish to run multiple apps for data with identical access controls (e.g., messages, photos, and docs in a project).

Secure multi-execution [33] provides non-interfering data containers for client-side code (one process per container, although faceted execution can relax that [24]). Self-protecting data [28] uses hardware IFC and a security policy component that does not protect against implicit information flows.

Maxoid [71] allows an app to give its sensitive data to an untrusted app while confining the untrusted app to not further leak the data. This functionality is equivalent to DATS's app component and similar to Hails plugins.

The IFC approaches above implement containers in different ways. DATS's declassification is applicable to all - it will safely remove declassifiable functionality from applications' TCB - while IFC can be applied to DATS's TCB itself.

\section{Conclusions}

DATS places applications out of the TCB while presenting a familiar security-oblivious programming model to developers. Extracting out access control from web services and enforcing it as a service is a major departure from current application-centric security models, but this is only one step forward towards placing users in control over their data. DATS motivates focusing language support for noninterference specifically towards template languages and architecture/OS support for multi-execution. In its current state, DATS is evidence that user privacy and developer productivity are not a zero-sum game.

\section{Acknowledgements}

The authors would like to thank Petros Maniatis, Dawn Song, and Krste Asanovic for key insights and the anonymous reviewers for their valuable feedback on earlier drafts of the work. This research was supported by the Israeli Ministry of Science, Technology and Space, and a collaboration grant through HiPEAC (European Union's H2020 research and innovation programme under grant agreement 687698). Lluís Vilanova was supported by an Andrew and Erna Finci Viterbi Post-Doctoral Fellowship. Charalampos Papamanthou was supported in part by NSF grants \#1652259 and \#1514261. 


\section{References}

[1] Advanced multi layered unification filesystem (AUFS). http://aufs. sourceforge.net.

[2] Celery. http://www.celeryproject.org.

[3] CVE Details. http://www.cvedetails.com/vulnerability-list.

[4] Docker. http://docker.com.

[5] Recent zero-day exploits. https://www.fireeye.com/current-threats/ recent-zero-day-attacks.html.

[6] Flask. http://flask.pocoo.org.

[7] Gitlab security vulnerabilities. https://www.cvedetails.com/ vulnerability-list/vendor_id-13074/Gitlab.html.

[8] 20 famous websites vulnerable to cross site scripting (XSS) attack. http://thehackernews.com/2011/09/20-famous-websitesvulnerable-to-cross.html.

[9] HITRUST alliance. https://hitrustalliance.net.

[10] Linux Containers. http://linuxcontainers.org.

[11] Mattermost security updates. https://about.mattermost.com/securityupdates/.

[12] Mustache. http://mustache.github.io.

[13] OWASP top ten project. https://www.owasp.org/index.php/OWASP_ Top_Ten_Project.

[14] React - a JavaScript library for building user interfaces. http://facebook. github.io/react.

[15] Redis. http://redis.io.

[16] Comparison of web template engines. https://en.wikipedia.org/wiki/ Comparison_of_web_template_engines (accessed Aug 2017).

[17] RFC 6455 - the websocket protocol. https://tools.ietf.org/html/rfc6455.

[18] Wikipedia - SQL Injection. https://en.wikipedia.org/wiki/SQL_ injection\#Examples.

[19] The security flaws at the heart of the Panama Papers. http://www.wired.co.uk/article/panama-papers-mossack-fonsecawebsite-security-problems.

[20] WSGI. http://wsgi.org.

[21] D. Akhawe, F. Li, W. He, P. Saxena, and D. Song. Data-confined HTML5 applications. In Computer Security - ESORICS, 2013.

[22] S. Arnautov, B. Trach, F. Gregor, T. Knauth, A. Martin, C. Priebe, J. Lind, D. Muthukumaran, D. O'Keeffe, M. L. Stillwell, D. Goltzsche, D. Eyers, R. Kapitza, P. Pietzuch, and C. Fetzer. SCONE: Secure Linux Containers with Intel SGX. In Symp. on Operating Systems Design and Implementation (OSDI), Nov. 2016.

[23] A. Askarov, D. Zhang, and A. C. Myers. Predictive black-box mitigation of timing channels. In ACM Conf. on Computer \& Communications Security (CCS), Oct. 2011.

[24] T. H. Austin and C. Flanagan. Multiple facets for dynamic information flow. Jan. 2012.

[25] A. Aviram, S.-C. Weng, S. Hu, and B. Ford. Efficient system-enforced deterministic parallelism. In Symp. on Operating Systems Design and Implementation (OSDI), Oct. 2012.

[26] S. Biswas, D. Franklin, A. Savage, R. Dixon, T. Sherwood, and F. T. Chong. Multi-execution: Multicore caching for data-similar executions. In Intl. Symp. on Computer Architecture (ISCA), June 2009.

[27] M. Castro, M. Costa, and J.-P. Martin. Better bug reporting with better privacy. In Intl. Conf. on Arch. Support for Programming Languages \& Operating Systems (ASPLOS), Mar. 2008.

[28] Y.-Y. Chen, P. A. Jamkhedkar, and R. B. Lee. A software-hardware architecture for self-protecting data. In ACM Conf. on Computer \& Communications Security (CCS), Oct. 2012.

[29] R. Cheng, W. Scott, P. Ellenbogen, J. Howell, F. Roesner, A. Krishnamurthy, and T. Anderson. Radiatus: Strong user isolation for scalable web applications. In ACM Symp. on Cloud Computing (SoCC), Oct. 2016.

[30] W. W.-Y. Cheng. Information Flow for Secure Distributed Applications. Ph.D., MIT, Cambridge, MA, USA, Aug. 2009. Also as Technical Report MIT-CSAIL-TR-2009-040.
[31] M. Dalton, H. Kannan, and C. Kozyrakis. Raksha: a flexible information flow architecture for software security. In Intl. Symp. on Computer Architecture (ISCA), May 2007.

[32] J. B. Dennis and E. C. V. Horn. Programming semantics for multiprogrammed computations. Comm. ACM, Mar. 1966.

[33] D. Devriese and F. Piersens. Noninterference through secure multiexecution. In IEEE Symp. on Security and Privacy, May 2010.

[34] C. Dwork. Differential privacy. In International Colloquium on Automata, Languages and Programming (ICALP), July 2006.

[35] P. Efstathopoulos, M. Krohn, S. VanDeBogart, C. Frey, D. Ziegler, E. Kohler, D. Mazières, F. Kaashoek, and R. Morris. Labels and Event Processes in the Asbestos Operating System. Oct. 2005.

[36] A. J. Feldman, A. Blankstein, M. J. Freedman, and E. W. Felten. Social networking with frientegrity: privacy and integrity with an untrusted provider. In USENIX Security Symposium, Aug. 2012.

[37] W. Felter, A. Ferreira, R. Rajamony, and J. Rubio. An updated performance comparison of virtual machines and linux containers. Technical report, IBM Research Division, July 2014.

[38] D. B. Giffin, A. Levy, D. Stefan, D. Terei, J. Mitchell, D. Mazières, and A. Russo. Hails: Protecting data privacy in untrusted web applications. In Symp. on Operating Systems Design and Implementation (OSDI), Oct. 2012 .

[39] J. A. Goguen and J. Meseguer. Security policies and security models. In IEEE Symp. on Security and Privacy, Apr. 1982.

[40] T. Hunt, Z. Zhu, Y. Xu, S. Peter, and E. Witchel. Ryoan: A distributed sandbox for untrusted computation on secret data. In Symp. on Operating Systems Design and Implementation (OSDI), Nov. 2016.

[41] Intel Software Guard Extensions Programming Reference. Intel, Oct. 2014.

[42] G. Klein, K. Elphinstone, G. Heiser, J. Andronick, D. Cock, P. Derrin, D. Elkaduwe, K. Engelhardt, R. Kolanski, M. Norrish, T. Sewell, H. Tuch, and S. Winwood. seL4: Formal verification of an OS kernel. In ACM Symp. on Operating Systems Principles (SOSP), Oct. 2009.

[43] M. Krohn. Information Flow Control for Secure Web Sites. PhD thesis, MIT, 2008.

[44] S. Lee, E. L. Wong, D. Goel, M. Dahlin, and V. Shmatikov. $\pi$ Box: A platform for privacy-preserving apps. In USENIX Symp. on Networked Systems Design and Implementation (NSDI), Apr. 2013.

[45] H. M. Levy. Capability-Based Computer Systems. Digital Press, 1984.

[46] N. Li, T. Li, and S. Venkatasubramanian. t-closeness: Privacy beyond k-anonymity and l-diversity. In Intl. Conf. on Data Engineering (ICDE), Apr. 2007.

[47] J. Liu, M. D. George, K. Vikram, X. Qi, L. Waye, and A. C. Myers. Fabric: a platform for secure distributed computation and storage. In $A C M$ Symp. on Operating Systems Principles (SOSP), Oct. 2009.

[48] P. Loscocco and S. Smalley. Integrating flexible support for security policies into the linux operating system. In USENIX Annual Technical Conf. (ATC), 2001.

[49] A. Machanavajjhala, D. Kifer, J. Gehrke, and M. Venkitasubramaniam. L-diversity: Privacy beyond k-anonymity. ACM Trans. Knowl. Discov. Data, Mar. 2007.

[50] F. McSherry. Privacy integrated queries. In SIGMOD, June 2009.

[51] P. Mohan, A. Thakurta, E. Shi, D. Song, and D. E. Culler. GUPT: Privacy preserving data analysis made easy. In SIGMOD, May 2012.

[52] A. Nadkarni, B. Andow, W. Enck, and S. Jha. Practical DIFC enforcement on Android. In USENIX Security Symposium, Aug. 2016.

[53] B. Parno, J. M. McCune, D. Wendlandt, D. G. Andersen, and A. Perrig. Clamp: Practical prevention of large-scale data leaks. In IEEE Symp. on Security and Privacy, May 2009.

[54] B. Parno, J. M. McCune, and A. Perrig. Bootstrapping trust in commodity computers. In IEEE Symp. on Security and Privacy, May 2010.

[55] V. Rastogi and S. Nath. Differentially private aggregation of distributed time-series with transformation and encryption. In SIGMOD, June 2010. 
[56] I. Roy, S. T. Setty, A. Kilzer, V. Shmatikov, and E. Witchel. Airavat: Security and privacy for mapreduce. In USENIX Symp. on Networked Systems Design and Implementation (NSDI), Apr. 2010.

[57] B. Russell. KVM and Docker LXC Benchmarking with OpenStack. http://bodenr.blogspot.com/2014/05/kvm-and-docker-lxcbenchmarking-with.html.

[58] A. Sabelfeld, A. C., and Myers. Language-based information-flow security. IEEE fournal on Selected Areas in Communications, Jan. 2003.

[59] M. Sherr and M. Blaze. Application containers without virtual machines. In ACM workshop on Virtual machine security, Nov. 2009.

[60] S. Smalley, C. Vance, and W. Salamon. Implementing SELinux as a Linux Security Module. Technical report, NAI Labs, Dec. 2001.

[61] D. Stefan, E. Z. Yang, P. Marchenko, A. Russo, D. Herman, B. Karp, and D. Mazières. Protecting users by confining javascript with COWL. In Symp. on Operating Systems Design and Implementation (OSDI), Oct. 2014.

[62] E. Stefanov, M. van Dijk, A. Juels, and A. Oprea. Iris: a scalable cloud file system with efficient integrity checks. In Annual Computer Security Applications Conference (ACSAC), Dec. 2010.

[63] L. Sweeney. k-anonimity: A model for protecting privacy. International fournal on Uncertaint, Fuzziness and Knowledge-based Systems, Oct. 2002.

[64] M. Tiwari, P. Mohan, A. Osheroff, H. Alkaff, E. Shi, E. Love, D. Song, and K. Asanović. Context-centric security. In Proceedings of the 7th USENIX Workshop on Hot Topics in Security, Aug. 2012.

[65] B. C. Vattikonda, S. Das, and H. Shacham. Eliminating fine grained timers in Xen (short paper). In Proceedings of CCSW 2011, Oct. 2011.

[66] L. Vilanova, M. Ben-Yehuda, N. Navarro, Y. Etsion, and M. Valero. CODOMs: Protecting software with code-centric memory domains. In Intl. Symp. on Computer Architecture (ISCA), June 2014.

[67] L. Vilanova, M. Jordà, N. Navarro, Y. Etsion, and M. Valero. Direct interprocess communication (dipc): Repurposing the codoms architecture to accelerate ipc. In European Conference on Computer Systems (EuroSys), Apr. 2017.
[68] D. Volpano, G. Smith, and C. Irvine. A sound type system for secure flow analysis. Journal of Computer Security, Sept. 2009.

[69] R. N. M. Watson, J. Anderson, B. Laurie, and K. Kennaway. Capsicum: practical capabilities for UNIX. In USENIX Security Symposium, Aug. 2010.

[70] R. N. M. Watson, J. Woodruff, P. G. Neumann, S. W. Moore, J. Anderson, D. Chisnall, N. Dave, B. Davis, K. Gudka, B. Laurie, S. J. Murdoch, R. Norton, M. Roe, S. Son, and M. Vadera. CHERI: A hybrid capabilitysystem architecture for scalable software compartmentalization. In IEEE Symp. on Security and Privacy, May 2015.

[71] Y. Xu and E. Witchel. Maxoid: Transparently confining mobile applications with custom views of state. In European Conference on Computer Systems (EuroSys), Apr. 2015.

[72] Y. Xu and E. Witchel. Earp: Principled storage, sharing, and protection for mobile apps. In USENIX Symp. on Networked Systems Design and Implementation (NSDI), Mar. 2016.

[73] Y. Xu, A. M. Dunn, O. S. Hofmann, M. Z. Lee, S. A. Mehdi, and E. Witchel. Application-defined decentralized access control. In USENIX Annual Technical Conf. (ATC), June 2014.

[74] J. Yang, K. Yessenov, and A. Solar-Lezama. A language for automatically enforcing privacy policies. Jan. 2012.

[75] A. Yip, X. Wang, N. Zeldovich, and M. F. Kaashoek. Improving application security with data flow assertions. In ACM Symp. on Operating Systems Principles (SOSP), Oct. 2009

[76] S. Zdancewic and A. C. Myers. Robust declassification. In IEEE Computer Security Foundations Workshop, June 2001.

[77] N. Zeldovich, S. Boyd-Wickizer, E. Kohler, and D. Mazières. Making Information Flow Explicit in HiStar. In Symp. on Operating Systems Design and Implementation (OSDI), Nov. 2006.

[78] N. Zeldovich, H. Kannan, M. Dalton, and C. Kozyrakis. Hardware enforcement of application security policies using tagged memory. In Symp. on Operating Systems Design and Implementation (OSDI), Dec. 2008.

[79] Y. Zhang, J. Katz, and C. Papamanthou. Integridb: Verifiable sql for outsourced databases. In ACM Conf. on Computer \& Communications Security (CCS), Oct. 2015. 